\title{
Overexpression of Osmyb4 enhances compatible solute accumulation and increases stress tolerance of Arabidopsis thaliana
}

\author{
Monica Mattana ${ }^{a, *}$, Elisa Biazzi ${ }^{a}$, Roberto Consonni ${ }^{b}$, Franca Locatelli ${ }^{a}$, Candida Vannini ${ }^{c}$, Stefano \\ Provera $^{\mathrm{d}}$ and Immacolata Coraggio ${ }^{\mathrm{a}}$ \\ astituto di Biologia e Biotecnologia Agraria, Consiglio Nazionale delle Ricerche, via Bassini 15, 20133 Milano, Italy \\ bIstituto per lo Studio delle Macromolecole, Laboratorio NMR, Consiglio Nazionale delle Ricerche, via Bassini, 15, 20133 Milano, Italy \\ 'Dipartimento Ambiente Salute e Sicurezza, Università dell'Insubria via J. H. Dunant 3, 21100 Varese, Italy \\ ${ }^{d}$ NMR Spectroscopy - Computational, Analytical and Structural Sciences GlaxoSmithKline Research Center via Fleming 4, 37135 Verona, Italy
}

\author{
Correspondence \\ *Corresponding author, \\ e-mail: mattana@ibba.cnr.it \\ Received 06 April 2005; revised 27 May 2005 \\ doi: 10.1111/j.1399-3054.2005.00551.x
}

In this paper, we report the metabolic and molecular changes in response to cold and drought induced in Osmyb4 transgenic Arabidopsis thaliana compared with the wildtype (WT). The rice Osmyb4 gene codes for a transcription factor (Myb4) induced by cold treatment and, in Arabidopsis transgenic plants, improves cold and freezing tolerance [Vannini C, Locatelli F, Bracale M, Magnani E, Marsoni M, Osnato M, Mattana M, Baldoni E, Coraggio I (2004) Plant J 37: 115-127]. Here, we report the ability of Myb4 to induce also drought tolerance in Arabidopsis transgenic plants. By the use of nuclear magnetic resonance (NMR) and enzymatic assays, we showed that several compatible solutes (glucose, fructose, sucrose, proline, glycine betaine and sinapoyl malate) accumulate in higher amount in Osmyb4-overexpressing plants with respect to the WT, both under normal and stress conditions. Considering proline, we also found that in transgenic plants the levels of the mRNAs coding for $\Delta^{1}$-pyrroline-5-carboxylate synthase (EC not assigned) and for $\Delta^{1}$-pyrroline-5-carboxylate dehydrogenase (EC 1.5.1.12) were higher and lower, respectively. The constitutive activation of several stress-inducible pathways and different kinetics in the accumulation of several metabolites, in Myb4 transgenic plants, may represent an advantage to prepare plants to face the stress condition. Moreover, these results taken together suggest that Myb4 integrates the activation of multiple components of stress response.

\section{Introduction}

Plants respond and adapt to abiotic stresses through various physiological and biochemical changes, including the expression of stress-specific genes (Bracale and Coraggio 2003, Bray 1997, Coraggio and Tuberosa 2004, Shinozaki and YamaguchiShinozaki 1997).
In particular, studies on cold-regulated gene expression established that the promoters of certain cold-responsive genes are activated by low temperature and dehydration stress (Dubouzet et al. 2003, Thomashow 1999). Studies on Arabidopsis led to the identification of a DNA regulatory element, the C-repeat (CRT)/dehydration responsive element (DRE), which imparts responsiveness to low

Abbreviations - LC-NMR, liquid chromatography-NMR; NMR, nuclear magnetic resonance; PAL, phenylalanine ammonia lyase; P5CDH, $\Delta^{1}$-pyrroline-5-carboxylate dehydrogenase; P5CS, $\Delta^{1}$-pyrroline-5-carboxylate synthase. 
temperature and drought (Yamaguchi-Shinozaki and Shinozaki 1994). Subsequently, five transcriptional activators were identified that bind to the CRT/DRE domain: three DREB1 and two DREB2 factors. The DREB1 factors are also known as CBF1, CBF2 and CBF3 (Gilmour et al. 1998, Liu et al. 1998, Shinwari et al. 1998, Stockinger et al. 1997). The CBF/DREB1 genes are rapidly induced in response to low temperature, whereas DREB2 factors are drought induced (Gilmour et al. 2000, Liu et al. 1998). Shinozaki and coworkers (Kasuga et al. 1999) showed that the CBF3/DREB1a overexpression in Arabidopsis plants results in increased tolerance not only to cold and freezing but also to dehydration stress caused by either drought or high salinity. The DREB proteins are probably the major transcription factors regulating cold-, high salt- and drought-inducible gene expression in Arabidopsis (Dubouzet et al., 2003). Recent studies (Dubouzet et al. 2003, Jaglo et al. 2001) indicate that the CBF cold-responsive pathway is conserved in Brassica napus and that components of the pathway are present in wheat and rye, which cold acclimate, as well as in tomato and rice, which do not.

In our laboratory, a rice gene, Osmyb4 (accession number Y11414), has been isolated and its role in cold acclimation has been elucidated by its CaMV35S-driven constitutive overexpression in Arabidopsis thaliana plants. Among transgenic plants, three different classes, depending on Osmyb4 gene expression, were defined: very low expression (VLE), medium expression (ME) and high expression (HE). The HE transgenic plants, like the CBF transgenic plants (Kasuga et al. 1999), exhibited a dwarf phenotype (Vannini et al. 2004). The expression of Osmyb4 gene in rice is induced by cold treatment $\left(4^{\circ} \mathrm{C}\right)$ but not by other stresses nor by the $\mathrm{ABA}$ hormone, indicating its involvement in the ABA-independent cold response. It has also been shown that Myb4 induces a dose-dependent cold and freezing tolerance in transgenic plants, possibly through the action of several metabolic stress-related pathways (Vannini et al. 2004).

The aim of the present study was to verify whether the transgenic plants were also drought tolerant and to compare some metabolic and molecular changes caused by cold and drought stresses in the HE transgenic plants with respect to the wildtype (WT), in control and stress conditions. Namely, we evaluated some parameters known to be important to confer tolerance to these kind of abiotic stresses. It is known that, as a result of drought and cold, plants accumulate a range of metabolically benign solutes, known as compatible solutes or osmolytes (Hare and Cress 1997, Hughes and Dunn 1996, Smirnoff 1998, Thomashow 1999, Xing and Rajashekar 2001). The compatible solutes we have focused on are proline and total amino acids, glycine betaine, soluble sugars and aromatic compounds. All these metabolites have been suggested to improve plant stress tolerance and it has been reported that the overexpression of proteins involved in their accumulation, such as a key enzyme for proline biosynthesis, produced a stress-tolerant phenotype (Dixon and Paiva 1995; Kishor et al. 1995, Shinozaki and YamaguchiShinozaki 1997, Wanner and Junttila 1999).

The quantitative determination of such metabolites has been performed by the combined use of enzymatic assay and ${ }^{1} \mathrm{H}$ nuclear magnetic resonance (NMR) in conjunction with LC-NMR (liquid chromatography-NMR) technique.

The data presented here show that the Osmyb4 transgenic plants are more drought-tolerant than the WT and suggest that cold and drought tolerance may depend on the Myb4 ability to integrate the activation of multiple components of the stress response.

\section{Materials and methods}

\section{Plant growth and treatments}

We used WT and transformed A. thaliana (ecotype Wassilewskija), constitutively expressing the Osmyb4 cDNA under the CaMV35S promoter (HE) (Vannini et al. 2004). After 3-day vernalization, seeds were placed in pots and seedlings were grown for 3 weeks with a 14/10-h photoperiod at $180 \mu \mathrm{M} \mathrm{m}^{-2} \mathrm{~s}^{-1}$, at $24^{\circ} \mathrm{C}$ during the day and $18^{\circ} \mathrm{C}$ at night. For coldacclimation experiments, plants were subjected to a continuous low-temperature $\left(4^{\circ} \mathrm{C}\right)$ treatment for different lengths of time: $8,24,48$ and $240 \mathrm{~h}$; the other conditions remaining unchanged. Plants were watered as needed during the cold treatment in order to prevent any water stress. For the analyses, the leaves were harvested and immediately frozen in liquid nitrogen.

For drought experiments 3-week-old, Arabidopsis plants were subjected to water withholding for 15 days. The leaf samples were collected after 5 and 9 days of drought treatment and immediately frozen in liquid nitrogen. After 15 days of drought treatment, a set of WT and HE plants (85 plants per each genotype) were watered and left 7 days to recover. Three independent experiments for each condition were performed. From each treatment (cold or drought), the plant samples were harvested separately to prepare RNA and extracts for enzymatic and NMR analyses.

For in vitro growth, vernalized seeds were sterilized and sown aseptically in 12-cm square petri dishes on MS agar medium. Two-week-old plants were laid down on $3 \mathrm{MM}$ paper sheet for $15 \mathrm{~min}$ to evaluate drought tolerance. Light and photoperiod conditions were the same as those used for soil-grown plants. 


\section{Soluble sugars, proline and amino acid measurements}

Proline and soluble sugars were extracted from the frozen leaves using $0.6 \mathrm{~N}$ perchloric acid, centrifuged at $16000 \mathrm{~g}$ for $15 \mathrm{~min}$ and the supernatant was used for the analysis. Proline concentration was determined as described previously (Vannini et al. 2004) according to the procedure of Bates et al. (1973). Proline content was calculated on the basis of a standard curve of L-Pro. The amino acid content was determined using the method of Moore (1968). Proline amounts were expressed both as $\mu \mathrm{g} \mathrm{m}^{-1}$ (fresh weight) FW and as proline to amino acids ratio.

On the same extracts, sucrose, glucose and fructose were quantified using the Boehringer Mannheim (RBiopharm, Darmstadt, Germany) kit, where the reduction of NADP was monitored at $340 \mathrm{~nm}$. Standard curves of sucrose, glucose and fructose were prepared.

\section{${ }^{1} \mathrm{H}$ NMR measurements}

\section{High-resolution NMR}

Arabidopsis leaf tissue was extracted with perchloric acid following the method of Menegus et al. (1988). After the extraction, the samples were lyophilized and then dissolved in $0.5 \mathrm{ml} \mathrm{D}{ }_{2} \mathrm{O}$. All ${ }^{1} \mathrm{H}$ spectra were acquired at 11.7 T on a Bruker Advance DMX spectrometer equipped with a $z$-gradient coil with a proton frequency of $500.13 \mathrm{MHz}$. Spectra were recorded at $300 \mathrm{~K}$, with a spectral width of $6000 \mathrm{~Hz}$ and 32-K data points and referenced to sodium trimethylsilyl $\left[2,2,3,3-{ }^{2} \mathrm{H} 4\right]$ propionate (TSP). Residual solvent suppression was achieved by applying a pre-saturation scheme with low-power radiofrequency irradiation. Bi-dimensional TOCSY experiments have been acquired for spin system assignment by using standard pulse sequence. All the spectra were analysed on PC equipped with the XWINNMR package (version 2.7, Bruker Biospin $\mathrm{GmbH}$, Rheinstetten/Karlsruhe, Germany). Each ${ }^{1} \mathrm{H}$ NMR spectrum was Fourier transformed without any resolution enhancement functions and corrected for phase and baseline distortions before integration of the selected resonances. Metabolite quantification has been performed by the use of external calibrated TSP capillary, inserted in each sample NMR tube.

\section{LC-NMR measurements}

$\mathrm{D}_{2} \mathrm{O} /$ acetonitrile $(0.1 \%$ trifluoroacetic acid) in 50/50 v/v was added to a lyophilized sample of the plant extract. The suspension was filtered in order to get a clean solution of extracted metabolites.
Varian Pro-Star 5 chromatograph with a Varian ProStar 330-diode array detector was used. One hundred microliter of metabolite mixture solution was injected onto a $150 \times 4.6-\mathrm{mm}$ Varian Polaris-A column. The flow rate was $1.0 \mathrm{ml}^{-1} \mathrm{~min}^{-1}$ with a gradient run starting from $5 \% \mathrm{ACN}$ up to $100 \% \mathrm{ACN}$ over $45 \mathrm{~min}$.

${ }^{1} \mathrm{H}$ NMR spectrum was acquired at $22^{\circ} \mathrm{C}$ on the chromatographic peak of interest at about 14 min using the stopped flow mode with Varian INOVA PFG $600-\mathrm{MHz}$ spectrometer operating at $14.09 \mathrm{~T}$, equipped with a Varian IFC probe (with a $60-\mu \mathrm{l}$ active volume flow cell) directly connected with the HPLC system. One thousand and twenty-four transients were collected over $12000 \mathrm{~Hz}$ of spectral width using $90^{\circ}$ pulses acquiring data into $32-\mathrm{K}$ points giving an acquisition time of $1.820 \mathrm{~s}$. WET saturation pulse sequence was applied in order to pre-saturate selectively both acetonitrile and residual water signals. The data were processed with a line broadening of $0.5 \mathrm{~Hz}$ to improve signal-tonoise ratio.

\section{RNA extraction and Northern blot analysis}

Total RNAs from WT and HE Arabidopsis plants were extracted with TRIzol Reagent (Invitrogen, Carlsbad, CA) following the manufacturer instructions. The RNAs were extracted from leaves of cold- and drought-treated Arabidopsis plants. Fifteen microgram of total RNA was loaded in a $1.2 \%$ agarose-formaldehyde gel and blotted onto Hybond- $\mathrm{N}^{+}$membrane (Amersham Biosciences, Piscataway, NJ). Hybridizations were performed with the Arabidopsis P5CS1 CDNA provided by Dr Szabados (Strizhov et al. 1997) and the Arabidopsis P5CDH complete cDNA provided by Dr Deuschle (Deuschle et al. 2001) labelled with a fluorescein labelling kit (Amersham Biosciences). Hybridizations and washes were performed under high stringency conditions $\left(0.2 \% \mathrm{SSC}\right.$ at $\left.65^{\circ} \mathrm{C}\right)$.

\section{Results}

\section{Plant tolerance to cold and drought stresses}

Because some genes involved in cold stress tolerance are known to follow a circadian rhythm (Kreps et al. 2002, Sauerbrunn and Schlaich 2004), all the Arabidopsis samples used for our analyses were harvested at 16:00 $\mathrm{h}$.

In a previous paper (Vannini et al. 2004), we reported that Osmyb4 overexpressing Arabidopsis plants increased their cold tolerance measured both considering physiological parameters and whole plant tolerance. In the present study, we found that Arabidopsis 
transgenic plants were more tolerant than the WT also to water deprivation (Fig. 1). As shown in Fig. 1A, the WT plants showed visible damage from the 9th day of drought treatment and, after 15 days, all plants died; the transgenic plants showed a better tolerance during all the time of treatment. Moreover, a 7-day-recovery experiment performed on 85 plants per genotype showed that a $30 \%( \pm 5)$ of the transgenic plants, but almost none $(1 \% \pm 1)$ of the WT, succeeded in growing and flowering (Fig. 1A).

The smaller (dwarf) phenotype of the HE plants (Vannini et al. 2004) could be in part responsible of

A
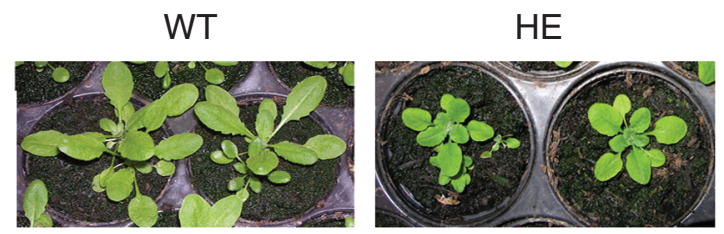

T9
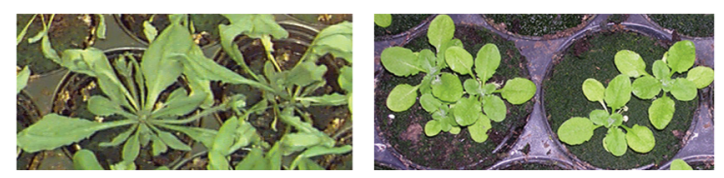

$\mathrm{T} 11$
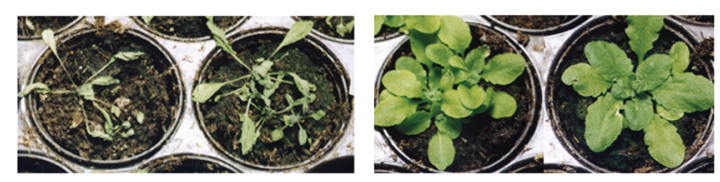

T15
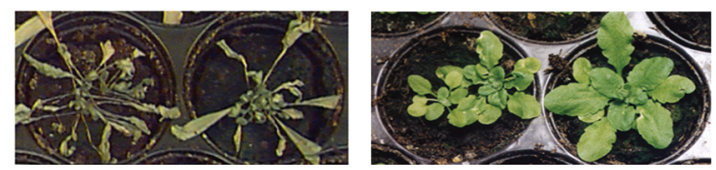

$\mathrm{R}$
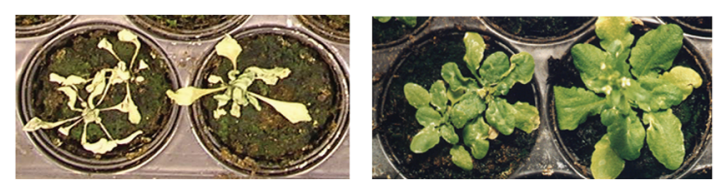

B
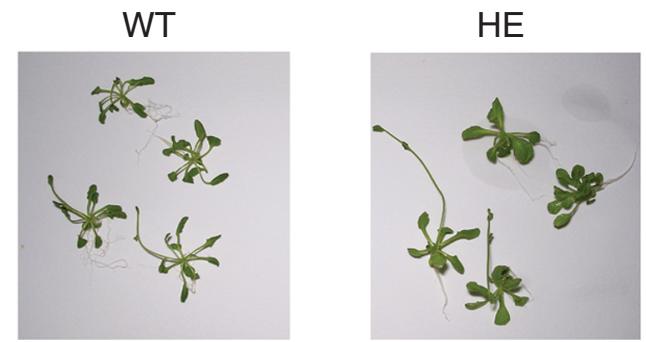

Fig. 1. Effect of drought treatment on wild-type (WT) and highexpression (HE) Arabidopsis. (A) The plants were grown for 3 weeks under normal conditions (TO) and then subjected to water withholding for 9,11 and 15 days (T9, T11 and T15, respectively). After 15 days, the plants were watered and left 7 days to recover (R). (B) The plants were grown for 2 weeks on MS agar medium, laid down on a 3MM paper sheet for $15 \mathrm{~min}$ and photographed. the drought tolerance. However, WT and HE in vitro grown plants show similar size. Therefore, drought tolerance was evaluated also on WT and HE in vitro grown plants. Two-week-old plantlets were laid down on a $3 \mathrm{MM}$ paper sheet and their water loss was phenotypically evaluated, and the plantlets were photographed after 15 min (Fig. 1B). As shown in Fig. 1B, WT plants were more wilted than the HE plants.

As expected, a cold treatment of 10 days $\left(4^{\circ} \mathrm{C}\right)$ did not cause any clear morphological damage either to the WT or to the transformed plants (data not shown).

\section{Proline content during cold and drought treatments}

It has been reported that proline accumulates in plants during adaptation to various types of environmental stresses such as drought, salinity, nutrient deficiency and cold (Delauney and Verma 1993, Hare et al. 1999, Ruiz et al. 2002). To follow the amount of proline during cold stress, we incubated 3-week-old Arabidopsis plants at $4^{\circ} \mathrm{C}$ for different lengths of time: $8,24,48$ and $240 \mathrm{~h}$. The concentration of proline was measured in leaf extracts and

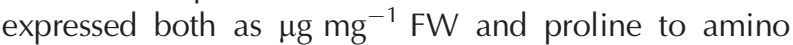
acids ratio. As shown in Fig. 2A, the concentration of proline increased in both genotypes during cold acclimation. However, in transgenic plants, proline content was higher than in WT during all the time courses. This difference between the two genotypes reached a peak after $48 \mathrm{~h}$ of cold (about three times higher than the WT). Even the proline to amino acids ratio increased during the cold treatment, being always higher in the transgenic lines, reaching about the double of the WT value at $48 \mathrm{~h}$ of cold treatment (Fig. 2A).

As far as the drought treatment is concerned, the concentration of proline was measured on leaves of plants left out watering for 5 and 9 days (Fig. 2B). After 11 days of drought, the WT plants were very damaged and were not sampled for the assay. After 5 days of drought, the proline content of the HE plants was three times higher than the WT. On the 9th day of drought, WT and transformed plants had a proline concentration very similar. When considering the proline to amino acids ratio, it was always higher in HE plants with respect to the WT with the higher difference at 5 days of drought (Fig. 2B).

\section{Transcript analysis for $\Delta^{1}$-pyrroline-5-carboxylate synthase and $\Delta^{1}$-pyrroline-5-carboxylate dehydrogenase}

Proline accumulation in response to different kind of stresses is correlated both with the transcriptional 
A
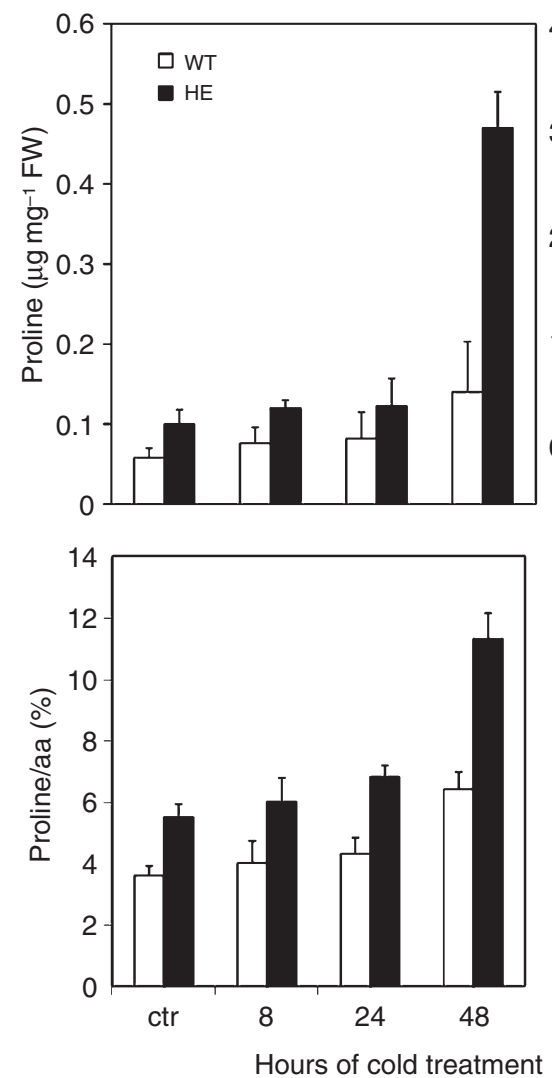

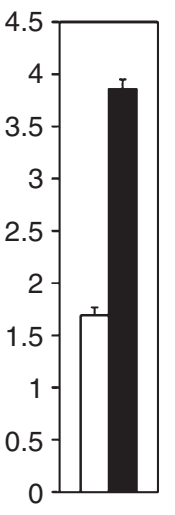

B
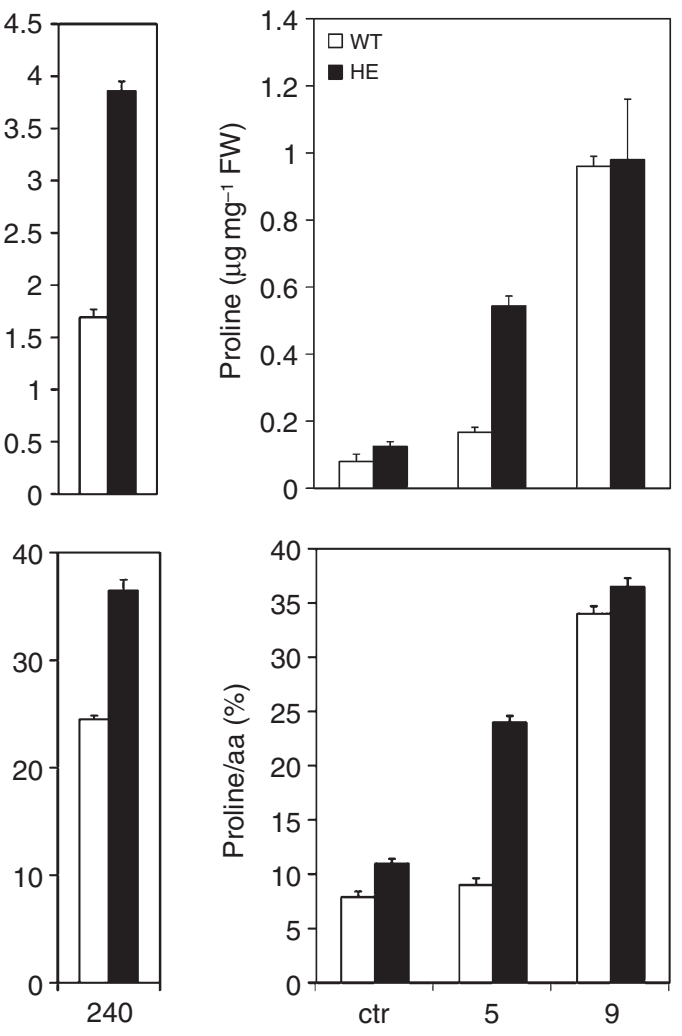

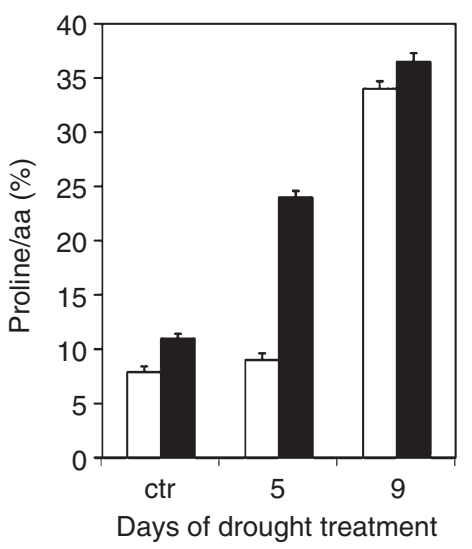

Fig. 2. Changes in the proline concentration in wild-type (WT) and high-expression (HE)

Arabidopsis leaves during cold and drought treatments. (A)

Three-week-old plants (control) were treated at $4^{\circ} \mathrm{C}$ for 8,24 , 48 and $240 \mathrm{~h}$ (B) Three-weekold plants were left out watering for 5 and 9 days. Concentration of proline is

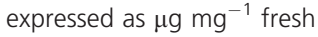
weight (FW) (upper panels). The proline/total amino acids (aa) ratio is shown at the lower panels. Results represent the mean of three independent experiments; bars represent standard deviations. activation of the gene encoding $\Delta^{1}$-pyrroline-5-carboxylate synthase (P5CS), the key regulatory and rate-limiting enzyme in the proline biosynthetic pathway, and with the inhibition of $\Delta^{1}$-pyrroline-5-carboxylate dehydrogenase $(\mathrm{P} 5 \mathrm{CDH})$, one of the two enzymes involved in proline catabolism (Delauney and Verma 1993, Strizhov et al. 1997). A Northern blot analysis on RNAs extracted from WT and HE Arabidopsis plants subjected to cold and drought stresses was performed. As shown in Fig. 3, a band of $2.4 \mathrm{~kb}$ corresponding to the transcript for P5CS was present in the WT- and HE-untreated plants (lanes 1, 2), being the level of transcript higher in HE. After $24 \mathrm{~h}$ of cold stress, the amount of transcript increased both in WT and HE, but it was much more abundant in HE plants (P5CS: lanes 3, 4).

The same analysis was carried out on mRNAs extracted from drought-treated plants. In this case, after 5 and 9 days of drought treatment, the amount of P5CS transcript increased both in WT and HE plants. In untreated and treated plants, the level of P5CS transcript was higher in transgenic plants than in the WT (P5CS: lanes 1, 2, 5, 6, 7 and 8).

The same filter was used for the detection of $P 5 C D H$ mRNA. Cold and drought treatments induced P5CDH transcription in both WT and HE plants. However, its amount was always higher in WT plants than in HE (Fig. 3; P5CDH: compare lanes 3, 5 and 7 with 4, 6 and 8 , respectively).

\section{Determination of free sugars}

We measured the accumulation of free sugars in WT and HE Arabidopsis leaves during cold and drought stresses using both an enzymatic method and a NMR analysis. By enzymatic assay, we found that the HEtransformed plants exhibited a higher concentration of glucose, fructose and sucrose with respect to the WT plants, both under cold and drought treatment (Fig. 4). The imposition of cold stress produced a general increase in sugar concentration in both genotypes (Fig. 4A). Moreover, the glucose and fructose amounts after 10 days at $4^{\circ} \mathrm{C}$ were reduced. In particular, WT glucose concentration was decreased to half of that after $48 \mathrm{~h}$, whereas the HE plants showed a $14 \%$ reduction only. The sucrose content during the acclimation experiment increased along with the time of treatment, being always higher in transformed plants than in the 

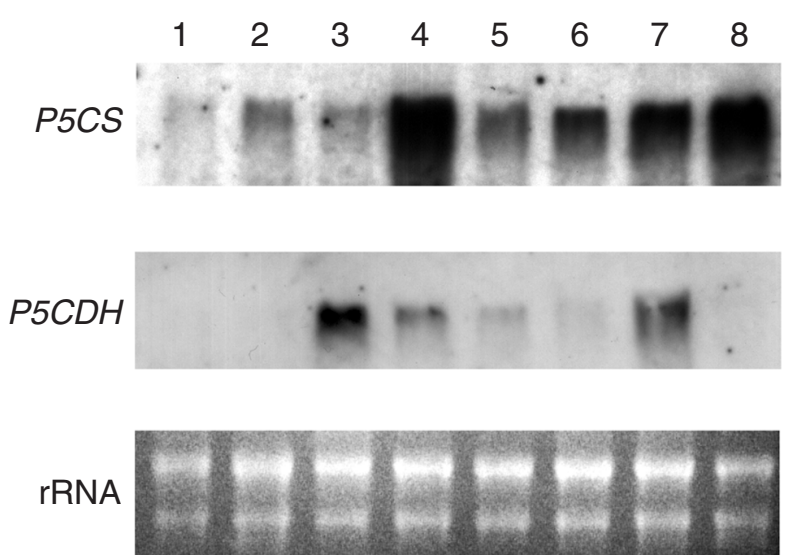

Fig. 3. Northern blot analysis of $P 5 C S$ and $P 5 C D H$ mRNA levels in coldand drought-treated leaves of wild-type (WT) and high-expression (HE) Arabidopsis plants. Fifteen microgram of total RNA was loaded in each lane. Lanes 1 and 2: untreated WT and HE plants; lanes 3 and 4: 24-h cold-treated WT and HE; lanes 5 and 6 : 5 days drought-treated WT and HE and lanes 7 and 8: 9 days drought-treated WT and HE. The same filter was used for hybridization with P5CS1 gene-specific probe and with $P 5 C D H$ gene specific-probe. Ethidium bromide-stained rRNA is shown below the RNA blots (rRNA).

WT. In particular, the sucrose level increased $60 \%$ after $240 \mathrm{~h}$ of cold stress (Fig. 4A).

NMR analysis performed on the WT- and HE-treated plants confirmed the data obtained by enzymatic assays (data not shown). Interestingly, NMR analysis highlighted the accumulation of other sugars besides those already enzymatically assayed. Indeed, in both WT and transgenic plants, the accumulation of this group of sugars increased during cold treatment, being always higher in the HE plants (Fig. 5A, dashed lines).

Under drought condition, the amount of the three sugars, enzymatically analysed, increased till 9 days of treatment. Also in this case, their levels in the HE were higher than in the WT; in particular, 9-day-treated HE plants accumulated 2.5-fold the amount of glucose and almost two-fold that of fructose of the WT (Fig. 4B).

\section{NMR analysis of metabolites}

By NMR analysis, we estimated the level of some osmolytes. Besides proline and sugars these compounds include some amino acids, glycine betaine and secondary metabolites (Dixon and Paiva 1995, Hare et al. 1998).

Alanine increases during cold acclimation both in WT and transgenic plants. The concentration of this amino acid did not differ in the two genotypes before the treatment, whereas after 10 days at $4^{\circ} \mathrm{C}$ was significantly higher in the transformed plants (Table 1).

The amount of glycine betaine in transgenic plants was twice the amount of the WT before the cold treatment. Both in WT and HE plants the glycine betaine content reached a maximum after $48 \mathrm{~h}$ of cold treatment, being 30\% higher in transformed plants (Table 1).

NMR analysis highlighted also interesting peaks in the aromatic region (Fig 5B; S). They all correspond to a single compound, which was identified as sinapoyl malate by LC-NMR analysis in combination with HPLC-NMR. In WT plants, its level doubled in 10 days of cold (Table 2). In the HE plants, it was present at a concentration 4-5 times higher than in the WT during all the time-course experiments (Table 2).

Analogous results were obtained in the case of drought experiments (data not shown).

Because the synapilic esters are known to be fluorescent (Chapple et al. 1992), we analysed the WT and the transgenic Arabidopsis plants under UV lamp and we could appreciate the fluorescence in roots of HE plants (Fig. 6).

\section{Discussion}

We previously reported the ability of the rice transcription factor Myb4 to confer chilling and freezing tolerance in transgenic Arabidopsis plants (Vannini et al. 2004). Considering that cold and drought stresses are both characterized by dehydration and because some transcription factors regulating gene expression in Arabidopsis are common to the two stresses (Dubouzet et al. 2003), we have studied the responses to both stresses in WT and HE plants.

We first assayed drought tolerance in Myb4-expressing plants compared with the WT. As shown in Fig. 1, transgenic plants showed a high degree of drought tolerance; actually, after 9 days of water deprivation, WT plants appeared already wilted, whereas Myb4expressing plants seemed undamaged up to 11 days. Moreover, after 15 days of water deprivation, followed by a week of recovery, all the WT plants died, whereas almost the $30 \%$ of transgenic plants survived. The experiments performed on in vitro grown plants indicated that the Myb4-driven drought tolerance is due to a specific functional effect, independent of the plant size (Fig. 1B). This result was confirmed by the finding that Osmyb4 overexpression increases drought tolerance also in other transformed species (apple, maize and tomato, Mattana et al. 2005). As well as HE Arabidopsis plants, the Osmyb4-transformed apple plants exhibit a dwarf phenotype. In this case, to assay drought tolerance, we determined the relative water content (RWC) of detached leaves of plants (WT and $\mathrm{HE}$ ) subjected to different periods of drought for up to 15 days. After 15 days of treatment (soil water potential $>-30 \mathrm{Mpa}$ ), a $15 \%$ reduction of the RWC 
A
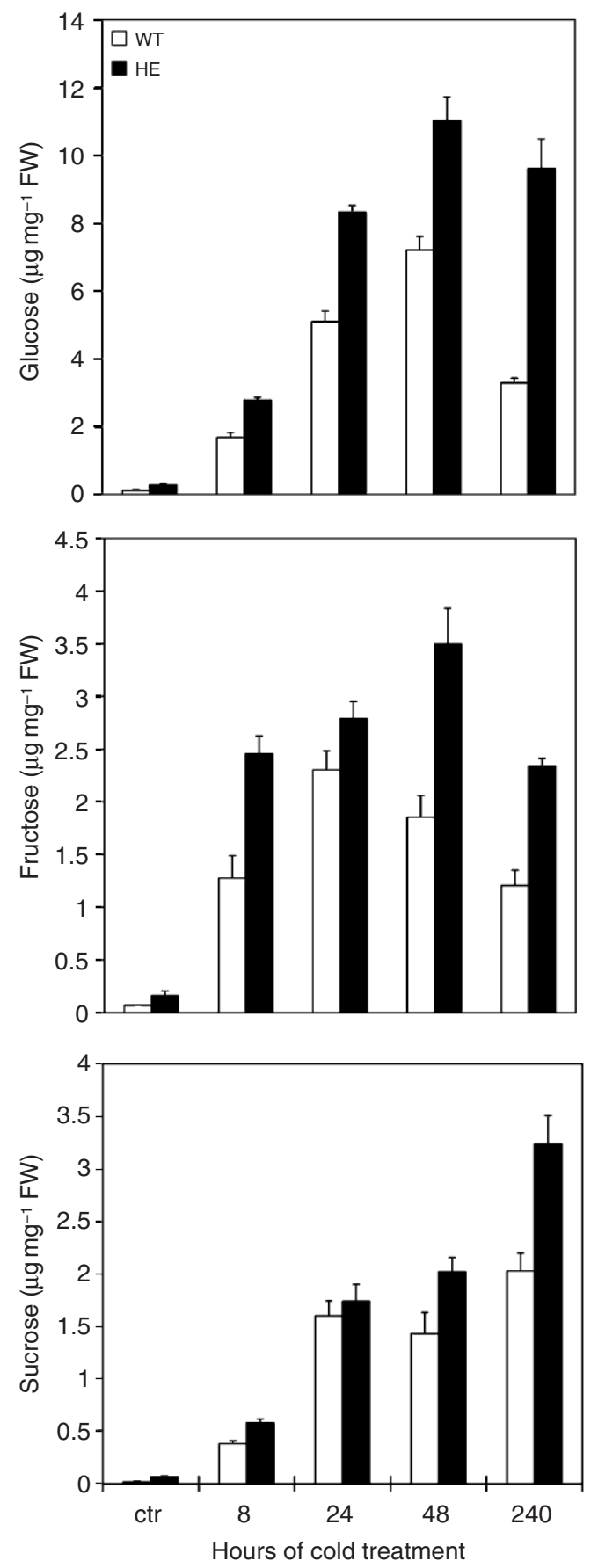

B
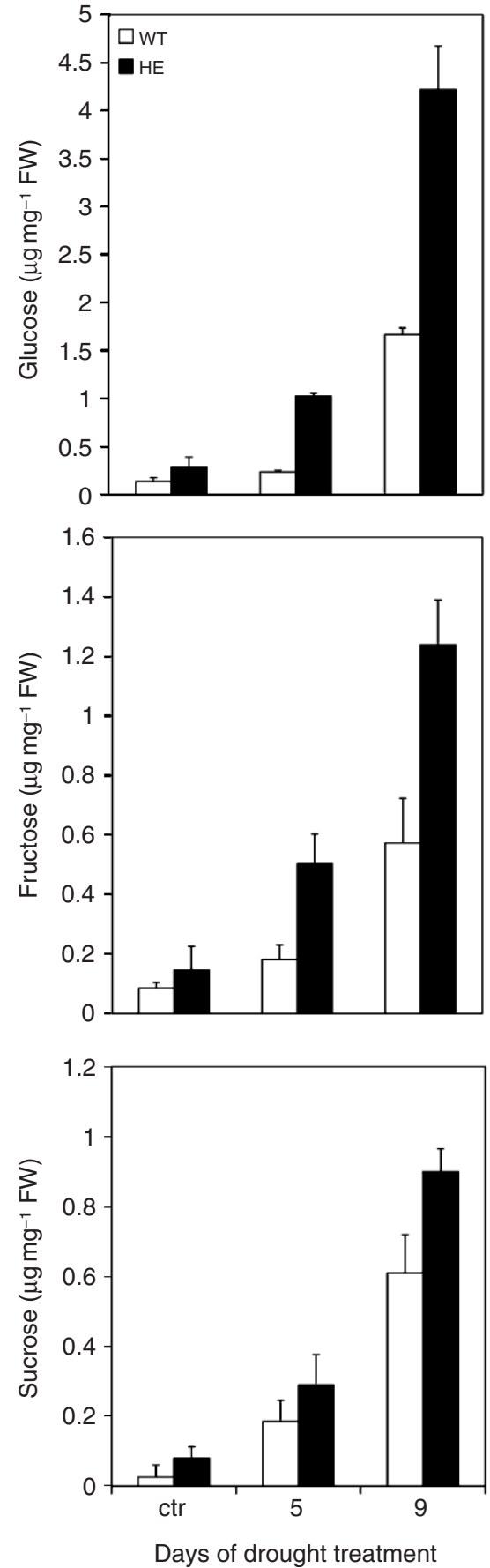

Fig. 4. Changes in the content of glucose, fructose and sucrose in wild-type (WT) and high-expression (HE)

Arabidopsis leaves during cold and drought treatments. (A)

Three-week-old Arabidopsis plants (control) were treated for $8,24,48$ and $240 \mathrm{~h}$ at $4^{\circ} \mathrm{C}$. (B) Three-week-old Arabidopsis plants (ctr) were subjected to water deprivation for 5 and 9 days. Concentration of glucose, fructose and sucrose is expressed as $\mu \mathrm{g} \mathrm{mg}^{-1}$ fresh weight (FW). Results represent the mean of three independent experiments, bars represent standard deviations. was detected for control plants, whereas in transgenic leaves no significant reduction was shown. Moreover, Osmyb4 strongly increases drought tolerance also in maize- and tomato-transformed plants without affecting their phenotype (Mattana et al. 2005).
We analysed also some metabolic changes induced by cold and drought stresses in WT $A$. thaliana compared with Osmyb4-transformed plants.

To this purpose, we followed the production of several metabolites known to improve stress tolerance, 
A

$240 \mathrm{~h}$
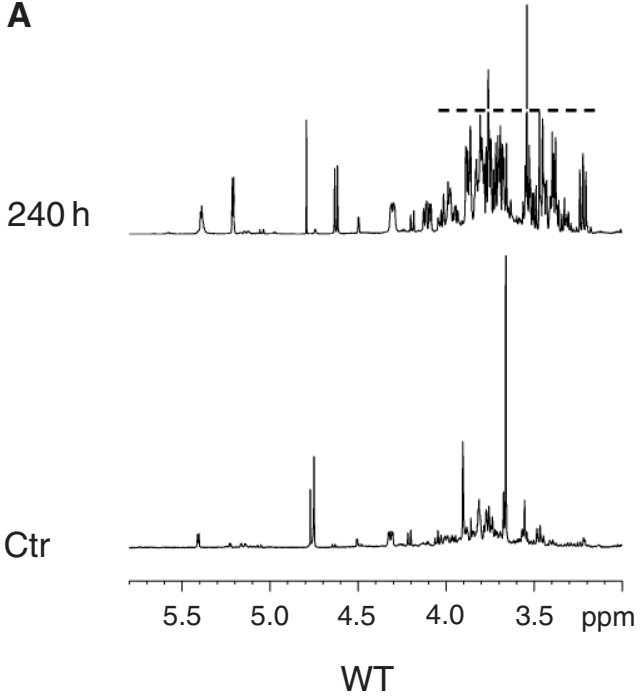

B

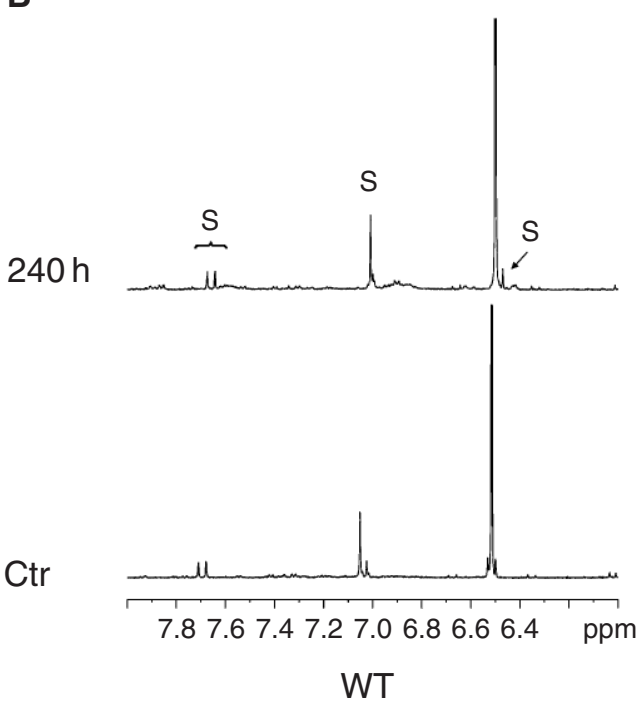

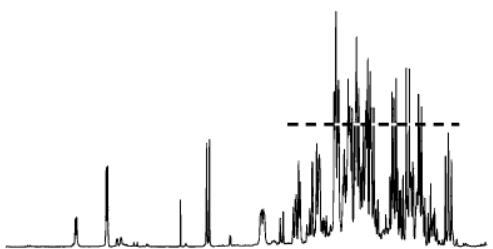

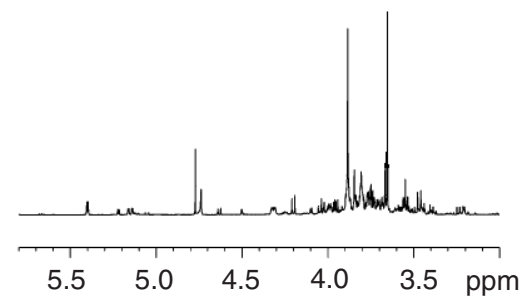

HE

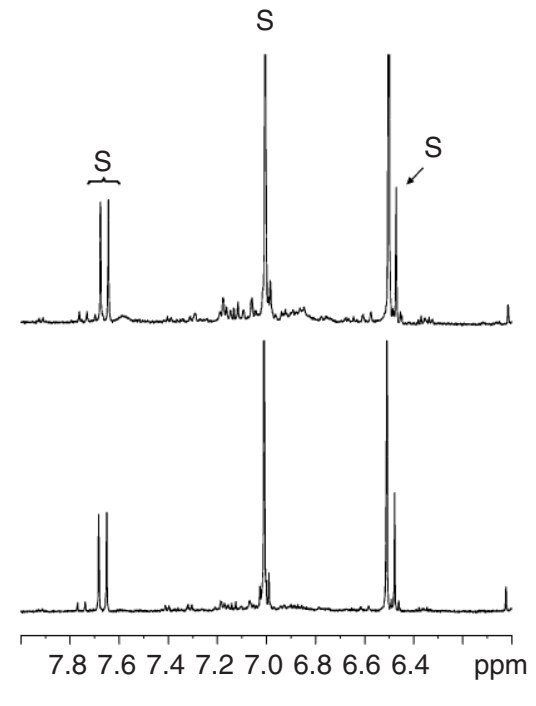

Fig. 5. ${ }^{1} \mathrm{H}$-nuclear magnetic resonance (NMR) spectra expansions of sugars and aromatic compounds extracted from cold-treated Arabidopsis wild-type (WT) and high-expression (HE) plants. (A) Sugar region: dashed lines summarize sidechain sugar protons. (B) Aromatic region: Sinapoyl-malate (S) resonance is indicated. using enzymatic and NMR approaches. We found that Osmyb4 transgenic plants contain a higher concentration of osmolytes even before the imposition of the stress. Actually, proline, sucrose, glucose, fructose and glycine betaine concentrations in HE plants were almost twice the amount of the WT before any stress treatment (Figs 2, 4 and 5 and Table 1). During the time course experiments, we observed that both cold and drought stresses induced the accumulation of the assayed solutes also in the WT plants but at lower concentration with respect to the transgenic lines (Figs 2, 4 and 5 and Table 1). In particular, the accumulation of free sugars in the form of glucose and sucrose is a well-documented mechanism by which plants acclimate to various stresses (Bonhert and Jensen 1996). In fact, these sugars can be quickly used for respiratory energy production upon return to more favourable conditions besides serving as osmoprotectants during the stress. Moreover, NMR analyses showed the increase also of other sugars during cold treatment most in transgenic than in WT plants, as highlighted by dashed lines in Fig. 5A. These sugars may belong to the raffinose family, as their accumulation under cold and drought stresses has been reported (Taji et al. 2002).

Glycine betaine, an amphoteric quaternary amine, plays an important role as compatible solute in plants under various stress, especially low temperature and drought (Sakamoto and Murata 2002). The molecular 
Table 1. Alanine and glycine betaine content in cold-treated wild-type (WT) and high-expression (HE) Arabidopsis plants quantified by ${ }^{1} \mathrm{H}$ NMR. Standard deviation was less than $3 \%$.

\begin{tabular}{|c|c|c|c|c|}
\hline \multirow[b]{2}{*}{ Time } & \multicolumn{2}{|c|}{ Alanine (ng $\mathrm{mg}^{-1} \mathrm{FW}$ ) } & \multicolumn{2}{|c|}{ Glycine betaine $\left(\mathrm{ng} \mathrm{mg}^{-1} \mathrm{FW}\right)$} \\
\hline & WT & $\mathrm{HE}$ & WT & $\mathrm{HE}$ \\
\hline Control & 94.42 & 100.70 & 5.38 & 11.45 \\
\hline $48 h$ & 552.17 & 737.19 & 118.85 & 154.88 \\
\hline $240 \mathrm{~h}$ & 560.16 & 865.74 & 94.48 & 113.51 \\
\hline
\end{tabular}

features of glycine betaine allow it to interact with macromolecules, stabilizing the structures and activities of enzymes and protein complexes (Xing and Rajashekar 2001). Plant species vary in their capacity to synthesize this compound, and Arabidopsis synthesizes glycine betaine at very low level in normal growth conditions (Xing and Rajashekar 2001). The accumulation of glycine betaine under cold stress (Table 1 ) shows an increase of this compound during $48 \mathrm{~h}$ of cold both in WT and HE plants, being always 30\% higher in the latter. Analogous results were observed during 5 days of drought treatment where the $\mathrm{HE}$ plants showed an increase of $40 \%$ with respect to the WT (data not shown). The inductive effect found in Osmyb4-overexpressing plants could be considerable, because it has been reported that even small accumulation of glycine betaine results in the induction of cold, freezing and drought tolerance (Rajashekar et al. 1999).

Accumulated proline has been proposed to protect enzymes and membranes during hostile environmental conditions (Hare and Cress 1997). In particular, proline is one of the compounds that act as compatible osmolytes to contrast the dehydration effects due to cold, freezing and drought stresses (Delauney and Verma 1993). Our data on proline accumulation in WT plants during cold and drought stresses (Fig. 2) are in agreement with those described in literature (Hare and Cress 1997, Hong et al. 2000). In particular, the HE transgenic plants exhibit an accumulation of proline higher than the WT under both unstressed and stressed conditions

Table 2. Sinapoyl malate content in cold-treated wild-type (WT) and high-expression (HE) Arabidopsis plants quantified by ${ }^{1} \mathrm{H}$ NMR. Standard deviation was less than $5 \%$.

\begin{tabular}{lll}
\hline & \multicolumn{2}{l}{ Sinapoyl malate $\left(\mathrm{ng} \mathrm{mg} \mathrm{mg}^{-1} \mathrm{FW}\right)$} \\
\cline { 2 - 3 } Time & WT & HE \\
\hline Control & 294.62 & 1146.35 \\
$48 \mathrm{~h}$ & 374.07 & 1397.20 \\
$240 \mathrm{~h}$ & 526.24 & 1478.29 \\
\hline
\end{tabular}

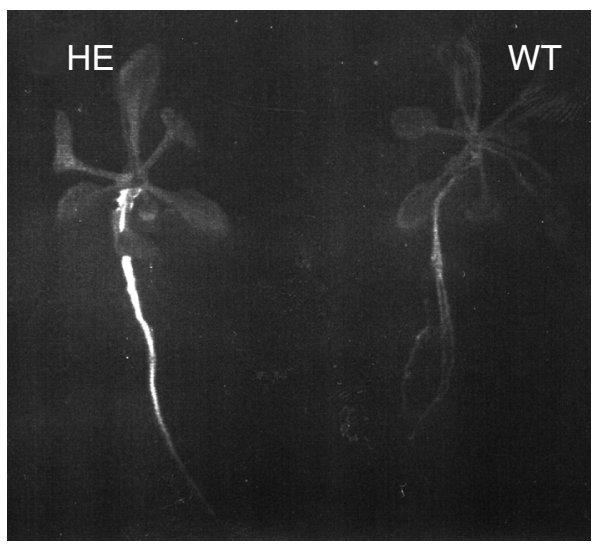

Fig. 6. Wild-type (WT) and Osmyb4 transgenic plants [high expression (HE)] photographed using a 312-nm transilluminator as a light source. The fluorescence of HE roots is due to the higher concentration of sinapoyl-malate.

(Fig. 2). However, after 9 days of drought the proline content in the two genotypes was very similar (Fig. 2). One possible explanation for this result may be the existence of a feedback mechanism controlling the proline synthesis. Moreover, the proline and other metabolite concentration in WT at that time were overestimated with respect to the $\mathrm{HE}$, for the higher degree of dehydration.

As the higher level of proline in HE plants might depend on its increased synthesis or its decreased degradation (Yoshiba et al., 1997), we analysed the mRNA coding for the enzymes catalysing the proline biosynthesis (P5CS) and the proline catabolism (P5CDH) (Fig. 3). The amount of the P5CS transcript found in the untreated HE plants was higher than in the WT; after cold stress, its level increased both in WT and transgenic plants, being much more abundant in the latter (Fig. 3, lanes 3 and 4). Analogous results were obtained in the case of drought stress (Fig. 3, lanes 5-8). These results are in agreement with those described in literature for stress-induced P5CS accumulation (Strizhov et al. 1997). The production of P5CDH mRNA in WT plants during cold and drought stresses was higher than in the HE-treated plants and increased along with the stress treatments (Fig. 3). The trend observed for P5CS and $P 5 C D H$ mRNAs reflects that observed for proline accumulation. To date, proline is considered one of the most important compatible solute because of its numerous metabolic functions during and after relief from the stress. This amino acid has been proposed to protect cellular structure during many kind of stresses, to detoxify free radicals by forming long-lived adducts with them and, during recovery, to generate high energy output through its degradation (Hare and Cress 1997, 
Rudolph et al. 1986). The fundamental role of proline in stress tolerance has been well demonstrated; indeed, the overproduction of this amino acid in crops leads to an increase of the overall environmental tolerance and thereby to an enhancement of the productivity (Hare and Cress 1997, Hong et al. 2000, Kishor et al. 1995).

It has been reported that many abiotic and biotic stresses induce phenylpropanoid accumulation (Dixon and Paiva 1995). All phenylpropanoids derive from cinnamic acid, which is formed from phenylalanine by the action of phenylalanine ammonia lyase (PAL). In a previous paper, we found that the expression of the PAL gene was enhanced in the HE plants (Vannini et al. 2004). The reported NMR data here suggest that the increased mRNA levels of $P A L$ and, possibly, of other genes involved in phenylpropanoid production result in a higher synthesis of aromatic compounds (Fig. 5B). In particular, we identified a highly accumulated aromatic compound like sinapoyl malate, one of the end metabolites of the phenylpropanoid pathway (Fig. 5B). The concentration of this compound was found to be four times higher in transgenic plants than in the WT in all assayed conditions (Table 2). The fluorescence of sinapoyl malate present in $\mathrm{HE}$ plants was revealed by the use of a 312-nm transilluminator (Fig. 6). UV irradiation induces flavonoids and sinapate esters in Arabidopsis (Dixon and Paiva 1995). These UV-absorbing compounds provide protection against UV-B damage and subsequent cell death by protecting DNA from dimerization and breakage. Namely, it has been reported that mutants lacking phenolic sunscreens or defective in sinapate esters synthesis are sensitive to UV irradiation (Jin et al. 2000, Landry et al. 1995). Actually, preliminary data we obtained on the UV stress tolerance indicate that in the HE plants this tolerance is strongly higher than in the WT, and it could be due to the great concentration of sinapoyl malate in the HE genotype.

The data obtained on the untreated WT and HE Arabidopsis plants indicate that the Osmyb4 overexpression constitutively activates several of the pathways usually induced in response to cold and drought stresses. On the basis of the results reported in this paper, we cannot discriminate between genes directly activated by Myb4 and those activated indirectly. Microarray analysis performed on WT and HE Arabidopsis plants showed that the expression of more than 250 genes is positively affected by the Osmyb4 overexpression (Vannini et al. submitted). Many of them encode for transcriptional factors and the $60 \%$ of their promoters cannot be bound by Myb4, indicating that Myb4 exerts its action on several genes indirectly (Vannini et al. submitted). However, the promoter in silico analysis suggested for some genes a direct Myb4 activation. In particular, in the P5CS1, but not in the P5CS2 promoter, multiple putative Myb4-binding sites are present. Moreover, we previously demonstrated the PAL promoter direct activation by Osmyb4 (Vannini et al. 2004).

The further increase in osmolyte concentration registered in the HE plants during the stress imposition indicates the existence of other pathways, Myb4-independent, specifically induced by the stress and/or the action of post-transcriptional regulation on some Myb4activated genes. The Myb4-driven improved stress tolerance may depend both on the higher level of osmolytes found in untreated plants, which prepares plants to face the stress, and on the larger amount of metabolites accumulated during the stress treatment. This kind of results are expected for the constitutive expression of an upstream acting transcriptional factor, and consistently, analogous results were reported for Arabidopsis plants constitutively overexpressing CBF3 (Gilmour et al. 2000). Also in this case, the authors report that transgenic plants show both a higher constitutive amount of proline and sugars and a different kinetic of their accumulation under stress conditions.

Acknowledgements - We thank Professor Laszlo Szabados (Hungarian Academy of Science) and Dr Karen Deuschle (Tubingen University, Germany) for the kind gifts of the Arabidopsis P5CS1 and P5CDH cDNAs, respectively. We thank Dr Eric de Combarieu, Research and Development Laboratories, Indena S.p.A. (Milan, Italy) for technical assistance in HPLC-MASS analysis. We also thank Dr Annamaria Genga for critical reading of the manuscript. This work was partially supported by the FIRB-strategic project postgenome, Program of the Italian Ministry of University and Scientific Research (code RBNE01LAC).

\section{References}

Bates LS, Waldren RP, Teare ID (1973) Rapid determination of free proline for water-stress studies. Plant Soil 39: 205-207

Bohnert HJ, Jensen RG (1996) Strategies for engineering waterstress tolerance in plants. Trends Biotechnol 14: 89-97

Bracale M, Coraggio I (2003) Chilling and freezing stresses in plants: cellular responses and molecular strategies for adaptation. In: Sanità di Toppi L, Pawlik-Skowronska B (eds) Abiotic Stresses in Plants. Kluwer Academic Publishers, Dordrecht, The Netherlands, pp 36-53

Bray EA (1997) Plant responses to water deficit. Trends Plant Sci 2: 48-54

Chapple CC, Vogt T, Ellis BE, Somerville CR (1992) An Arabidopsis mutant defective in the general phenylpropanoid pathway. Plant Cell 4: 1413-1424 
Coraggio I, Tuberosa R (2004) Molecular bases of plant adaptation to abiotic stresses and approaches to enhance tolerance to hostile environments. In: Christou P, Klee H (eds) Handbook of Plant Biotechnology, Vol. 1. John Wiley and Sons Limited Publishers, Chichester, UK, pp 413-468

Delauney AJ, Verma DPS (1993) Proline biosynthesis and osmoregulation in plants. Plant J 4: 215-223

Deuschle K, Funck D, Hellmann H, Daschner K, Binder S, Frommer WB (2001) A nuclear gene encoding mitochondrial $\Delta^{1}$-pyrroline-5-carboxylate dehydrogenase and its potential role in protection from proline toxicity. Plant J 27: 345-355

Dixon RA, Paiva NL (1995) Stress-induced phenylpropanoid metabolism. Plant Cell 7: 1085-1097

Dubouzet JG, Sakuma Y, Ito Y, Kasuga M, Dubouzet EG, Miura S, Seki M, Shinozaki K, Yamaguchi-Shinozaki K (2003) OsDREB genes in rice, Oryza sativa L., encode transcription activators that function in drought-, high-saltand cold-responsive gene expression. Plant J 33: 751-763

Gilmour SJ, Zarka DG, Stockinger EJ, Salazar MP, Houghton JM, Thomashow MF (1998) Low temperature regulation of the Arabidopsis CBF family of AP2 transcriptional activators as an early step in cold-induced COR gene expression. Plant J 16: 433-442

Gilmour SJ, Sebolt AM, Salazar MP, Everard JD, Thomashow MF (2000) Overexpression of the Arabidopsis CBF3 transcriptional activator mimics multiple biochemical changes associated with cold acclimation. Plant Physiol 124: 1854-1865

Hare PD, Cress WA (1997) Metabolic implications of stressinduced proline accumulation in plants. Plant Growth Regul 21: 79-102

Hare PD, Cress WA, Van Staden J (1998) Dissecting the roles of osmolyte accumulation during stress. Plant Cell Environ 21: 535-553

Hare PD, Cress WA, Van Staden J (1999) Proline synthesis and degradation: a model system for elucidating stressrelated signal transduction. J Exp Bot 50: 413-434

Hong Z, Lakkineni K, Zhang Z, Verma DPS (2000) Removal of feedback inhibition of $\Delta^{1}$-Pyrroline-5-carboxylate synthetase results in increased proline accumulation and protection of plants from osmotic stress. Plant Physiol 122: 1129-1136

Hughes MA, Dunn MA (1996) The molecular biology of plant acclimation to low temperature. J Exp Bot 47: 291-305

Jaglo KR, Kleff S, Amundsen KL, Zhang X, Haake V, Zhang JZ, Deits T, Thomashow MF (2001) Components of the Arabidopsis C-repeat/dehydration-responsive element binding factor cold-response pathway are conserved in Brassica napus and other plant species. Plant Physiol 127: 910-917

Jin H, Cominelli E, Bailey P, Parr A, Mehrtens F, Jones J, Tonelli C, Weisshaar B, Martin C (2000) Transcriptional repression by AtMYB4 controls production of UV-protecting sunscreens in Arabidopsis. EMBO J 19: 6150-6161

Kasuga M, Liu Q, Miura S, Yamaguchi-Shinozaki K, Shinozaki K (1999) Improving plant drought, salt, and freezing tolerance by gene transfer of a single stressinducible transcription factor. Nature Biotechnol 17: 287-291

Kishor PBK, Hong Z, Miao G-H, Hu C-AA, Verma DPS (1995) Overexpression of $\Delta^{1}$-pyrroline 5-carboxylate synthetase increases proline production and confers osmotolerance in transgenic plants. Plant Physiol 108: 1387-1394

Kreps JA, Wu Y, Chang H-S, Zhu T, Wang X, Harper JF (2002) Transcriptome changes for Arabidopsis in response to salt, osmotic, and cold stress. Plant Physiol 130: 2129-2141

Landry LG, Chapple CC, Last RL (1995) Arabidopsis mutants lacking phenolic sunscreens exhibit enhanced ultraviolet-B injury and oxidative damage. Plant Physiol 109: 1159-1166

Liu Q, Kasuga M, Sakuma Y, Abe H, Miura S, YamaguchiShinozaki K, Shinozaki K (1998) Two transcription factors, DREB1 and DREB2, with an EREBP/AP2 DNA-binding domain separate two cellular signal transduction pathways in drought- and low temperature responsive gene expression, respectively. Arabidopsis. Plant Cell 10: 1391-1406

Mattana M, Carravieri S, Vannini C, Bracale M, Locatelli F, Baldoni E, Pasquali G, Mancuso S, Biricolti S, Natoli V, Corneti S, Tuberosa R, Laura M, Allavena A, Faoro F, Iriti M, Coraggio I (2005) Osmyb4: a tool to improve multiple stress tolerance in crops. In: Evenson RE, Santaniello V (eds) Agricultural Biotechnology: Ten Years After, 9th ICABR International Conference, in press

Menegus F, Cattaruzza L, Chersi A, Selva A, Fronza G (1988) Production and organ distribution of succinate in rice seedlings during anoxia. Physiol Plant 74: 444-449

Moore S (1968) Amino acid analysis: aqueous dimethyl sulfoxide as solvent for the ninhydrin reaction. J Biol Chem 243: 6281-6283

Rajashekar CB, Zhou H, Marcum KB, Prakash O (1999) Glycine betaine accumulation and induction of cold tolerance in strawberry (Fragaria X ananassa Duch.) plants. Plant Sci 148: 175-183

Rudolph AS, Crowe JH, Crowe LM (1986) Effects of three stabilising agents - proline, betaine and trehalose - on membrane phospholipids. Arch Biochem Biophys 245: 134-143

Ruiz JM, Sanchez E, Garcia PC, Lopez-Lefebre LR, Rivero RM, Romero L (2002) Proline metabolism and NAD kinase activity in greenbean plants subjected to cold-shock. Phytochemistry 59: 473-478

Sakamoto A, Murata N (2002) The role of glycine betaine in the protection of plants from stress: clues from transgenic plants. Plant Cell Environ 25: 163-171 
Sauerbrunn N, Schlaich NL (2004) PCC1: a merging point for pathogen defence and circadian signalling in Arabidopsis. Planta 218: 552-561

Shinozaki K, Yamaguchi-Shinozaki K (1997) Gene expression and signal transduction in water-stress response. Plant Physiol 115, 327-334

Shinwari ZK, Nakashima K, Miura S, Kasuga M, Seki M, Yamaguchi-Shinozaki K, Shinozaki K (1998) An Arabidopsis gene family encoding DRE/CRT-binding proteins involved in low-temperature-responsive gene expression. Biochem Biophys Res Commun 250: 161-170

Smirnoff N (1998) Plant resistance to environmental stress. Curr Opin Biotechnol 9: 214-219

Stockinger EJ, Gilmour SJ, Thomashow MF (1997) Arabidopsis thaliana CBF1 encodes an AP2 domaincontaining transcriptional activator that binds to the C-repeat/DRE, a cis-acting DNA regulatory element that stimulates transcription in response to low temperature and water deficit. Proc Natl Acad Sci USA 94: 1035-1040

Strizhov N, Abraham E, Okresz L, Blickling S, Zilberstein A, Schell J, Koncz C, Szabados L (1997) Differential expression of two P5CS genes controlling proline accumulation during salt-stress requires $\mathrm{ABA}$ and is regulated by $A B A 1$, $A B I 1$ and $A X R 2$ in Arabidopsis. Plant J 12: 557-569

Taji T, Ohsumi C, luchi S, Seki M, Kasuga M, Kobayashi M, Yamaguchi-Shinozaki K, Shinozaki K (2002) Important role of drought- and cold-inducible genes for galactinol synthase in stress tolerance in Arabidopsis thaliana. Plant J 29: 417-426

Thomashow MF (1999) Plant cold acclimation: freezing tolerance genes and regulatory mechanisms. Annu Rev Plant Physiol Plant Mol Biol 50: 571-599

Vannini C, Locatelli F, Bracale M, Magnani E, Marsoni M, Osnato M, Mattana M, Baldoni E, Coraggio I (2004) Overexpression of the rice Osmyb4 gene increases chilling and freezing tolerance of Arabidopsis thaliana plants. Plant J 37: 115-127

Vannini C, Iriti M, Bracale M, Locatelli F, Faoro F, Croce P, Coraggio I, Genga A (2005) The ectopic effect of the rice Osmyb4 gene in transgenic plants increases tolerance to abiotic and biotic stresses. (Submitted)

Wanner LA, Junttila O (1999) Cold-induced freezing tolerance in Arabidopsis. Plant Physiol 120: 391-399

Xing W, Rajashekar CB (2001) Glycine betaine involvement in freezing tolerance and water stress in Arabidopsis thaliana. Environ Exp Bot 46: 21-28

Yamaguchi-Shinozaki K, Shinozaki K (1994) A novel cisacting element in an Arabidopsis gene is involved in responsiveness to drought, low-temperature, or high-salt stress. Plant Cell 6: 251-264

Yoshiba Y, Kiyosue T, Nakashima K, Yamaguchi-Shinozaki K, Shinozaki K (1997) Regulation of levels of proline as an osmolyte in plants under water stress. Plant Cell Physiol 38: 1095-1102 